\title{
Chern-Simons supergravity on supergroup manifolds
}

\section{Castellani, ${ }^{a, b, c}$ C.A. Cremonini ${ }^{d, e}$ and P.A. Grassi ${ }^{a, b, c}$}

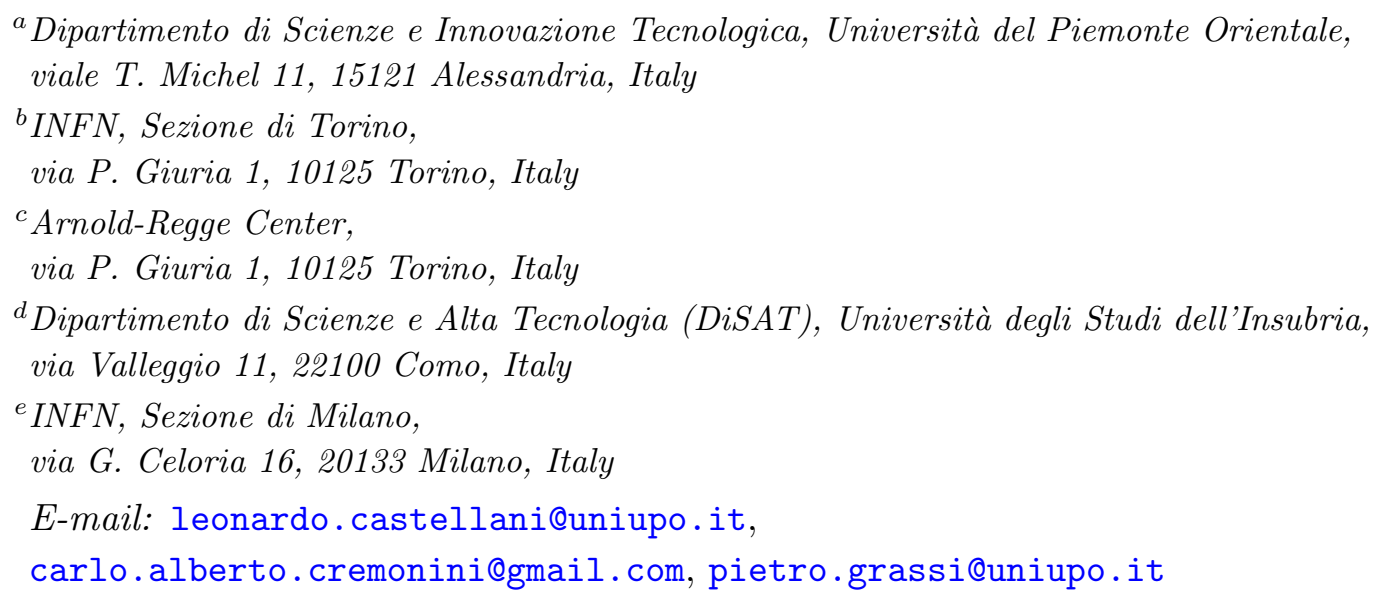

ABSTRACT: We construct $\mathrm{N}=1 \mathrm{~d}=3 \mathrm{AdS}$ supergravity within the group manifold approach and compare it with Achucarro-Townsend Chern-Simons formulation of the same theory. We clarify the relation between the off-shell super gauge transformations of the ChernSimons theory and the off-shell worldvolume supersymmetry transformations of the group manifold action. We formulate the Achucarro-Townsend model in a double supersymmetric action where the Chern-Simons theory with a supergroup gauge symmetry is constructed on a supergroup manifold. This framework is useful to establish a correspondence of degrees of freedom and auxiliary fields between the two descriptions of $d=3$ supergravity.

Keywords: Chern-Simons Theories, Superspaces

ARXIV EPRINT: 2002.09400 


\section{Contents}

1 Introduction 1

$2 \quad N=1, d=3$ AdS supergravity as Chern-Simons 2

$3 \quad N=1, d=3$ AdS supergravity in the group geometric approach $\quad 4$

3.1 The Lagrangian 5

3.2 Action and symmetries 5

3.3 Curvature parametrizations and symmetries of the spacetime action 6

4 Off-shell $N=1, d=3$ AdS supergravity $\quad 8$

4.1 Off-shell degrees of freedom 8

4.2 The extended superAdS algebra 8

$\begin{array}{lll}4.3 & \text { Curvature parametrizations } & 8\end{array}$

$\begin{array}{ll}4.4 & \text { The Lagrangian }\end{array}$

4.5 Off-shell supersymmetry transformations 9

$\begin{array}{ll}4.6 & \text { Field equations } \\ & 10\end{array}$

5 Equivalence of transformations: trivial gauge transformations $\quad 10$

6 Supersymmetric Achucarro-Townsend model 13

$\begin{array}{llr}7 & \text { Supersymmetry } & 16\end{array}$

8 Conclusions and outlook $\quad 18$

$\begin{array}{lr}\text { A Gamma matrices in } D=3 & \mathbf{1 8}\end{array}$

$\begin{array}{lll}\text { A.1 Useful identities } & 18\end{array}$

$\begin{array}{lll}\text { A.2 } & \text { Fierz identity for two Majorana one-forms } & 19\end{array}$

\section{Introduction}

We consider the $N=1$ anti-de Sitter supergravity action in $d=3$, realized as the difference of two Chern-Simons actions [1], with respectively $\operatorname{OSp}(1 \mid 2)$ and $\operatorname{Sp}(2)$ connections. Starting from the Chern-Simons formulation, we derive the supergravity action following the steps of the Achucarro and Townsend construction. One obtains a theory whose fundamental 1-form fields are (after a simple redefinition) the dreibein $V^{a}$, the spin connection $\omega^{a b}$ and the Majorana gravitino $\psi$. The action is invariant by construction under the gauge transformations of $\operatorname{OSp}(1 \mid 2) \otimes \operatorname{Sp}(2)$. The transformations generated by the spinorial (Majorana) charge of the supergroup yield the $N=1$ supersymmetry transformations, and close off-shell without need of auxiliary fields since they are really part of a gauge algebra. 
The action, being the integral of a 3 -form on a 3 -dimensional manifold, is also invariant by construction under $3 \mathrm{~d}$ diffeomorphisms. The latter are distinct from the gauge symmetries generated by the translation charges of the gauge supergroup.

Next we consider the (super)group-geometric construction of $N=1, d=3$ anti-de Sitter supergravity [2]. In this framework the basic 1-form fields live on the whole supergroup manifold $\operatorname{OSp}(1 \mid 2) \otimes \operatorname{Sp}(2)$, and the spacetime coordinates are identified with the parameters of the translation subgroup. Here supersymmetry is realized as a diffeomorphism in this supergroup manifold, in the fermionic directions. We will call it worldvolume supersymmetry to distinguish it from the gauge supersymmetry of the Chern-Simons action (see [3, 4], see also [5] where the difference between gauge symmetries and diffeomorphisms was studied for supergravity actions). To obtain a spacetime action (involving fields that depend only on spacetime coordinates), so as to be able to compare it with the Achucarro-Townsend action, it is necessary to integrate out the dependence on the Lorentz and Grassmann coordinates of the supermanifold. The resulting spacetime action coincides with the Achucarro and Townsend action, and is worldvolume supersymmetric provided some conditions are fulfilled, called "rheonomic" conditions. We show how these conditions can be imposed as constraints on the "outer" (i.e. along Grassmann differentials) components of the 2 -form curvatures, and how this leads to a local supersymmetry that not surprisingly coincides with the gauge supersymmetry. Here the origin of supersymmetry is geometric, whereas the gauge supersymmetry of the Chern-Simons action is totally algebraic. A peculiarity of the $d=3$ theory is that there exist additional symmetries, due to other possible rheonomic conditions, that close only on-shell. In particular the action is also invariant under a supersymmetry that has an extra term in the spin connection transformation with respect to the gauge supersymmetry of the CS action.

Supergravity in $d=3 \mathrm{AdS}$ spacetime can also be formulated with an additional bosonic auxiliary field, to balance off-shell degrees of freedom (the superspace formulation can be found in [6-8]). In this formulation we find that worldvolume supersymmetry does not require all curvatures to be horizontal in the Grassmann directions, and that Bianchi identities are satisfied off-shell. The resulting theory extends the Achucarro-Townsend action with terms depending on the auxiliary field. Once the auxiliary field is eliminated via its (algebraic) field equation, the Achucarro-Townsend action is recovered.

\section{$2 \quad N=1, d=3$ AdS supergravity as Chern-Simons}

Here we treat the simplest $N=1$ case. We consider therefore the difference between a CS action for $\operatorname{OSp}(1 \mid 2)$ and a $\mathrm{CS}$ action for $\operatorname{Sp}(2)$ :

$$
S=\kappa \int_{M^{3}} \operatorname{STr}\left(\mathbf{R} \boldsymbol{\Omega}+\frac{1}{3} \boldsymbol{\Omega}^{3}\right)-\kappa \int_{M^{3}} \operatorname{Tr}\left(\widetilde{\mathbf{R}} \widetilde{\boldsymbol{\Omega}}+\frac{1}{3} \widetilde{\boldsymbol{\Omega}}^{3}\right)
$$

where the 1 -form $\operatorname{OSp}(1 \mid 2)$ and $\operatorname{Sp}(2)$ connections are given respectively by the $3 \times 3$ supermatrix $\boldsymbol{\Omega}$ and the $2 \times 2$ matrix $\widetilde{\boldsymbol{\Omega}}$ :

$$
\boldsymbol{\Omega}=\left(\begin{array}{cc}
A^{a} \gamma_{a} & \frac{1}{\sqrt{\lambda}} \psi \\
\frac{i}{\sqrt{\lambda}} \bar{\psi} & 0
\end{array}\right), \quad \widetilde{\boldsymbol{\Omega}}=\widetilde{A}^{a} \gamma_{a}
$$


and the 2-form curvatures are

$$
\mathbf{R}=d \boldsymbol{\Omega}-\boldsymbol{\Omega} \wedge \boldsymbol{\Omega} \equiv\left(\begin{array}{cc}
R^{a}(A) \gamma_{a} & \frac{1}{\sqrt{\lambda}} \Sigma \\
\frac{i}{\sqrt{\lambda}} \bar{\Sigma} & 0
\end{array}\right), \quad \widetilde{\mathbf{R}}=\widetilde{R}^{a}(\widetilde{A}) \gamma_{a}
$$

with

$$
\begin{aligned}
R^{a}(A) & =d A^{a}+\varepsilon^{a}{ }_{b c} A^{b} A^{c}-\frac{1}{2 \lambda} \bar{\psi} \gamma^{a} \psi \\
\widetilde{R}^{a}(\widetilde{A}) & =d \widetilde{A}^{a}+\varepsilon^{a}{ }_{b c} \widetilde{A}^{b} \widetilde{A}^{c} \\
\Sigma & =d \psi-A^{a} \gamma_{a} \psi \\
\bar{\Sigma} & =d \bar{\psi}-\bar{\psi} \gamma_{b} A^{b}
\end{aligned}
$$

Carrying out the traces in (2.1) leads to the action

$$
S=2 \kappa \int_{M^{3}} R^{a}(A) A_{a}-\widetilde{R}^{a}(\widetilde{A}) \widetilde{A}_{a}-\frac{1}{3}\left(A^{a} A^{b} A^{c}-\widetilde{A}^{a} \widetilde{A}^{b} \widetilde{A}^{c}\right) \varepsilon_{a b c}-\bar{\psi} \Sigma
$$

Defining now the dreibein $V^{a}$ and the spin connection $\omega^{a b}$ as combinations of the $A^{a}$ and $\widetilde{A}^{a}$ connections:

$$
A^{a}=\frac{1}{2}\left(\omega^{a}+\frac{1}{\lambda} V^{a}\right), \quad \widetilde{A}^{a}=\frac{1}{2}\left(\omega^{a}-\frac{1}{\lambda} V^{a}\right)
$$

the action (2.8) becomes:

$$
S=-\frac{\kappa}{\lambda} \int_{M^{3}}\left(\mathcal{R}^{a b} V^{c} \varepsilon_{a b c}-\frac{1}{3 \lambda^{2}} V^{a} V^{b} V^{c}+2 i \bar{\psi} \Sigma\right)
$$

where

$$
\mathcal{R}^{a b} \equiv d \omega^{a b}-\omega^{a}{ }_{c} \omega^{c b}
$$

is the Lorentz curvature, and the gravitino curvature $\Sigma$ is expressed as

$$
\Sigma=d \psi-\frac{1}{4} \omega^{a b} \gamma_{a b} \psi-\frac{1}{2 \lambda} V^{a} \gamma_{a} \psi
$$

Symmetries. The action (2.1) or equivalently (2.10) is invariant (up to boundary terms) under the gauge transformations:

$$
\begin{array}{llll}
\delta_{\varepsilon} \boldsymbol{\Omega}=d \varepsilon-\boldsymbol{\Omega} \varepsilon+\varepsilon \boldsymbol{\Omega}, & & \Rightarrow & \delta_{\varepsilon} \mathbf{R}=-\mathbf{R} \varepsilon+\varepsilon \mathbf{R} \\
\delta_{\widetilde{\varepsilon}} \widetilde{\boldsymbol{\Omega}}=d \widetilde{\boldsymbol{\varepsilon}}-\widetilde{\boldsymbol{\Omega}} \widetilde{\varepsilon}+\widetilde{\boldsymbol{\varepsilon}} \widetilde{\boldsymbol{\Omega}}, & \Rightarrow & \delta_{\widetilde{\varepsilon}} \widetilde{\mathbf{R}}=-\widetilde{\mathbf{R}} \widetilde{\boldsymbol{\varepsilon}}+\widetilde{\boldsymbol{\varepsilon}} \widetilde{\mathbf{R}}
\end{array}
$$

where $\varepsilon$ and $\widetilde{\varepsilon}$ are the $\operatorname{OSp}(1 \mid 2)$ and $\operatorname{Sp}(2)$ gauge parameters:

$$
\varepsilon=\left(\begin{array}{cc}
\eta^{a} \gamma_{a} & \frac{1}{\sqrt{\lambda}} \epsilon \\
i \\
\frac{i}{\sqrt{\lambda}} \bar{\epsilon} & 0
\end{array}\right), \quad \widetilde{\varepsilon}=\widetilde{\eta}^{a} \gamma_{a}
$$

On the component fields the gauge transformations (2.13) and (2.14) take the form:

$$
\begin{aligned}
\delta A^{a} & =d \eta^{a}+2 A^{b} \eta^{c} \varepsilon_{b c}^{a}+\frac{i}{\lambda} \bar{\epsilon} \gamma^{a} \psi \\
\delta \widetilde{A}^{a} & =d \widetilde{\eta}^{a}+2 A^{b} \widetilde{\eta}^{c} \varepsilon^{a}{ }_{b c} \\
\delta \psi & =d \epsilon-A^{a} \gamma_{a} \epsilon+\eta^{a} \gamma_{a} \psi
\end{aligned}
$$


Using now the definitions (2.9), the gauge transformations on the supergravity fields read:

$$
\begin{aligned}
\delta V^{a} & =\mathcal{D} \varepsilon^{a}+\varepsilon_{b}^{a} V^{b}+i \bar{\epsilon} \gamma^{a} \psi \\
\delta \omega^{a b} & =\mathcal{D} \varepsilon^{a b}-\frac{2}{\lambda^{2}} V^{[a} \varepsilon^{b]}-\frac{i}{\lambda} \bar{\epsilon} \gamma^{a b} \psi \\
\delta \psi & =\mathcal{D} \epsilon-\frac{1}{2 \lambda} V^{a} \gamma_{a} \epsilon+\frac{1}{4} \varepsilon^{a b} \gamma_{a b} \psi+\frac{1}{2 \lambda} \varepsilon^{a} \gamma_{a} \psi
\end{aligned}
$$

The translation and Lorentz rotation parameters $\varepsilon^{a}$ and $\varepsilon^{a b}$ are defined in terms of $\eta^{a}$ and $\widetilde{\eta}^{a}$ as

$$
\varepsilon^{a} \equiv \lambda\left(\eta^{a}-\widetilde{\eta}^{a}\right), \quad \varepsilon^{a b} \equiv \varepsilon_{c}^{a b}\left(\eta^{c}+\widetilde{\eta}^{c}\right)
$$

and $\mathcal{D}$ is the Lorentz covariant derivative:

$$
\begin{aligned}
\mathcal{D} \varepsilon^{a} & \equiv d \varepsilon^{a}-\omega^{a}{ }_{b} V^{b} \\
\mathcal{D} \varepsilon^{a b} & \equiv d \varepsilon^{a b}-\omega^{a}{ }_{c} \varepsilon^{c b}+\omega^{b}{ }_{c} \varepsilon^{c a} \\
\mathcal{D} \epsilon & \equiv d \epsilon-\frac{1}{4} \omega^{a b} \gamma_{a b} \epsilon
\end{aligned}
$$

Field equations. Varying the action (2.10) in $V^{a}, \omega^{a b}$ and $\psi$ leads to the field equations:

$$
\begin{aligned}
\mathcal{R}^{a b}-\frac{1}{\lambda^{2}} V^{a} V^{b}+\frac{i}{2 \lambda} \bar{\psi} \gamma^{a b} \psi & =0 \\
\mathcal{D} V^{a}-\frac{i}{2} \bar{\psi} \gamma^{a} \psi & =0 \\
\mathcal{D} \psi-\frac{1}{2 \lambda} V^{a} \gamma_{a} \psi & =0
\end{aligned}
$$

The left hand sides are the curvatures of the $\operatorname{OSp}(1 \mid 2) \times \operatorname{Sp}(2)$ supergroup, in the rotated basis $\left(V^{a}, \omega^{a b}, \psi\right)$. These equations in fact are just the Cartan-Maurer equations of the supergroup, and are the starting point of the group-geometric construction of next section.

\section{$3 \quad N=1, d=3$ AdS supergravity in the group geometric approach}

The $\operatorname{OSp}(1 \mid 2) \times \operatorname{Sp}(2)$ Cartan-Maurer equations yield the definitions of the super-AdS curvatures:

$$
\begin{aligned}
R^{a b} & =d \omega^{a b}-\omega^{a}{ }_{c} \omega^{c b}-\frac{1}{\lambda^{2}} V^{a} V^{b}+\frac{i}{2 \lambda} \bar{\psi} \gamma^{a b} \psi \\
R^{a} & =d V^{a}-\omega^{a}{ }_{b} V^{b}-\frac{i}{2} \bar{\psi} \gamma^{a} \psi \equiv \mathcal{D} V^{a}-\frac{i}{2} \bar{\psi} \gamma^{a} \psi \\
\Sigma & =d \psi-\frac{1}{4} \omega^{a b} \gamma_{a b} \psi-\frac{1}{2 \lambda} V^{a} \gamma_{a} \psi=\mathcal{D} \psi-\frac{1}{2 \lambda} V^{a} \gamma_{a} \psi
\end{aligned}
$$

The Cartan-Maurer equations are invariant under the rescalings

$$
\omega^{a b} \rightarrow \omega^{a b}, V^{a} \rightarrow u V^{a}, \psi \rightarrow u^{\frac{1}{2}} \psi, \lambda \rightarrow u \lambda
$$

Taking exterior derivatives of both sides yields the Bianchi identities:

$$
\begin{aligned}
\mathcal{D} R^{a b}+\frac{2}{\lambda^{2}} R^{[a} V^{b]}+\frac{i}{\lambda} \bar{\psi} \gamma^{a b} \Sigma & =0 \\
\mathcal{D} R^{a}+R_{b}^{a} V^{b}-i \bar{\psi} \gamma^{a} \Sigma & =0 \\
\mathcal{D} \Sigma+\frac{1}{4} R^{a b} \gamma_{a b} \psi+\frac{1}{2 \lambda} R^{a} \gamma_{a} \psi-\frac{1}{2 \lambda} V^{a} \gamma_{a} \Sigma & =0
\end{aligned}
$$




\subsection{The Lagrangian}

Applying the building rules of the geometric approach [9-11] yields the Lagrangian 3-form

$$
\begin{aligned}
L & =R^{a b} V^{c} \varepsilon_{a b c}+2 i \bar{\psi} \Sigma+\frac{2}{3 \lambda^{2}} V^{a} V^{b} V^{c}-\frac{i}{2 \lambda} \bar{\psi} \gamma^{a b} \psi V^{c} \varepsilon_{a b c}= \\
& =\mathcal{R}^{a b} V^{c} \varepsilon_{a b c}-\frac{1}{3 \lambda^{2}} V^{a} V^{b} V^{c}+2 i \bar{\psi} \Sigma
\end{aligned}
$$

and is formally identical to the Achucarro-Townsend Lagrangian of the previous section. Note however that in the present framework this 3-form lives on the whole $N=1$ superspace $M^{3 \mid 2}$. It is obtained by considering the most general Lorentz scalar 3-form, given in terms of the super AdS curvatures and fields, invariant under the rescalings discussed above, and such that the variational equations admit the vanishing curvatures solution

$$
R^{a b}=R^{a}=\Sigma=0
$$

\subsection{Action and symmetries}

The action is now an integral over the whole superspace $M^{3 \mid 2}$

$$
S=\int_{M^{3 \mid 2}} L \wedge \eta_{M^{3}}
$$

and $\eta_{M^{3}}$ is the Poincaré dual of the 3-dimensional Minkowski space $M^{3}$ immersed into the superspace $\mathcal{M}^{(3 \mid 2)}$ (see e.g. [12-15]). $\eta_{M^{3}}$ is a 2 -form in superspace ${ }^{1}$ that after integration localizes the Lagrangian on the $d=3$ bosonic subspace, i.e.

$$
S=\int_{M^{3}} L_{\theta=d \theta=0}
$$

The action then exactly reproduces the Achucarro-Townsend spacetime action. Written as in (3.10) the action is automatically invariant under superdiffeomorphisms in superspace, since it is a 5-form integrated on a 5-dimensional superspace. The superdiffeomorphisms along a tangent vector $v$ in superspace act on the 1-form fields in the Lagrangian $L$ with the Lie derivative $\ell_{v} \equiv d \iota_{v}+\iota_{v} d$, i.e.

$$
\begin{aligned}
\delta_{v} V^{a} & =d\left(\iota_{v} V^{a}\right)+\iota_{v} d V^{a}=d\left(\iota_{v} V^{a}\right)+\iota_{v} R^{a}+\iota_{v}\left(\omega_{b}^{a} V^{b}\right)+\frac{i}{2} \iota_{v}\left(\bar{\psi} \gamma_{a} \psi\right) \\
& =\mathcal{D}\left(\iota_{v} V^{a}\right)+\iota_{v} R^{a}+\iota_{v}\left(\omega_{b}^{a}\right) V^{b}+\frac{i}{2} \iota_{v}\left(\bar{\psi} \gamma_{a} \psi\right)
\end{aligned}
$$

and similarly

$$
\begin{aligned}
\delta_{v} \omega^{a b} & =\mathcal{D}\left(\iota_{v} \omega^{a b}\right)+\iota_{v} R^{a b}+\frac{1}{\lambda^{2}} \iota_{v}\left(V^{a} V^{b}\right)-\frac{i}{2 \lambda} \iota_{v}\left(\bar{\psi} \gamma^{a b} \psi\right) \\
\delta_{v} \psi & =\mathcal{D}\left(\iota_{v} \psi\right)+\iota_{v} \Sigma+\frac{1}{4} \iota_{v}\left(\omega^{a b}\right) \gamma_{a b} \psi+\frac{1}{2 \lambda} \iota_{v}\left(V^{a} \gamma_{a} \psi\right)
\end{aligned}
$$

\footnotetext{
${ }^{1} \eta_{M^{3}}$ is really a $(0 \mid 2)$-integral form in superspace and the Lagrangian has to be considered as a $(3 \mid 0)$ superform so that the whole integrand is a $(3 \mid 2)$ top form in superspace.
} 
These are the built-in invariances of the action (3.10). Here resides most of the power of the group manifold formalism: if one considers the "mother" action (3.10) on $M^{3 \mid 2}$, the guaranteed symmetries are all the diffeomorphisms on $M^{3 \mid 2}$, generated by the Lie derivative $\ell_{v}$ along the tangent vectors $v$ of $M^{3 \mid 2}$. But how do these symmetries transfer to the spacetime action (3.11)?

The variation of the superspace action under diffeomorphisms generated by $\ell_{v}$ is ${ }^{2}$

$$
\delta S=\int_{M^{3 \mid 2}} \ell_{v}\left(L \wedge \eta_{M^{3}}\right)=\int_{M^{3 \mid 2}}\left(\ell_{v} L\right) \wedge \eta_{M^{3}}+L \wedge \ell_{v} \eta_{M^{3}}=0
$$

modulo boundary terms. One has to vary the fields ${ }^{3}$ in $L$ as well as the submanifold embedded in $M^{3 \mid 2}$ : the sum of these two variations gives zero ${ }^{4}$ on the superspace action $S$. But what we need in order to have a spacetime interpretation of all the symmetries of $S$, is really

$$
\delta S=\int_{M^{3 \mid 2}}\left(\ell_{v} L\right) \wedge \eta_{M^{3}}=0
$$

If this holds, varying the fields $\phi$ inside $L$ with the Lie derivative $\ell_{v}$ as in (3.12)-(3.14), and then projecting on spacetime $(\theta=0, d \theta=0)$, yields spacetime variations

$$
\delta \phi(\theta=0, d \theta=0))=\left.\ell_{v} \phi(x, \theta)\right|_{\theta=0, d \theta=0)}
$$

that leave the spacetime action (3.11) invariant. We call them spacetime invariances. They originate from the diffeomorphism invariance of the group manifold action, and give rise to symmetries of the spacetime action (3.11) only when (3.16) holds. This happens when $\ell_{v} L$ is exact, since $\eta$ is closed [2]. Exactness of $\ell_{v} L$ is equivalent to the condition

$$
\iota_{v} d L=d \alpha
$$

The Lagrangian $L$ depends on the basic fields $V^{a}, \omega^{a b}, \psi$ and their AdS curvatures $R^{A}=R^{a}, R^{a b}, \Sigma$ so that also $d L$, using the Bianchi identities, is expressed in terms of the fields and their curvatures. Then condition (3.18) translates into conditions on the contractions $\iota_{v} R^{A}$, i.e. conditions on the curvature components. In the jargon of the group-geometric approach, these are called "rheonomic" conditions, and must be consistent with Bianchi identities. The symmetry transformations of the theory are then given by equations (3.12)-(3.14), where the contractions $\iota_{v} R^{a}, \iota_{v} R^{a b}, \iota_{v} \Sigma$ are replaced by their expressions given by the rheonomic conditions.

\subsection{Curvature parametrizations and symmetries of the spacetime action}

Computing the exterior derivative of the Lagrangian in (3.8) and using the Bianchi identities (3.5)-(3.7) yields:

$$
d L=R^{a b} R^{c} \varepsilon_{a b c}+2 i \bar{\Sigma} \Sigma
$$

\footnotetext{
${ }^{2}$ Recall that $\ell_{v}$ (top form $)=d\left(\iota_{v}\right.$ top form $)$.

${ }^{3}$ Since $\ell_{v}$ satisfies the Leibniz rule, $\ell_{v} L$ can be computed by varying in turn all fields inside $L$.

${ }^{4}$ In the following the vanishing of action variations will always be understood modulo boundary terms.
} 
The condition

$$
i_{\epsilon} d L=d \alpha
$$

where $\epsilon$ is a tangent vector in fermionic directions is satisfied if all curvatures have no "legs" in fermionic directions, i.e. if $i_{\epsilon} R^{A}=0$. This leads to the parametrizations of the curvatures

$$
\begin{aligned}
R^{a b} & =R^{a b}{ }_{c d} V^{a} V^{b} \\
R^{a} & =R_{b c}^{a} V^{b} V^{c} \\
\Sigma & =\Sigma_{a b} V^{a} V^{b}
\end{aligned}
$$

and the transformations generated by the Lie derivative along the supergroup directions are:

$$
\begin{aligned}
\delta V^{a} & =\delta_{\text {gauge }} V^{a}+2 \varepsilon^{b} R_{b c}^{a} V^{c} \\
\delta \omega^{a b} & =\delta_{\text {gauge }} \omega^{a b}+2 \varepsilon^{c} R_{c d}^{a b} V^{d} \\
\delta \psi & =\delta_{\text {gauge }} \psi+2 \varepsilon^{a} \Sigma_{a b} V^{b}
\end{aligned}
$$

where $\delta_{\text {gauge }}$ are the gauge variations of the Achucarro - Townsend action given in (2.19)(2.21). The difference are terms proportional to the AdS curvatures: these terms are necessary for the transformation parametrized by $\varepsilon^{a}$ to be a spacetime diffeomorphism, rather than a gauge translation.

The spacetime reduced action being equal to the Achucarro-Townsend action, it also has its gauge symmetries. These coincide with the ones expressed by eqs. (3.24)-(3.26), except for gauge translations, that are an additional symmetry. In other words, the action is invariant under the CS gauge symmetry (2.19)-(2.21), and ordinary spacetime diffeomorphisms.

But there is an additional symmetry, due to another solution of (3.20), provided by the parametrizations:

$$
\begin{aligned}
R^{a b} & =R_{c d}^{a b} V^{c} V^{d}+\bar{\theta}_{c}^{a b} \psi V^{c} \\
R^{a} & =0 \\
\Sigma & =\Sigma_{a b} V^{a} V^{b}
\end{aligned}
$$

with

$$
\bar{\theta}_{c}^{a b}=c_{1} \bar{\Sigma}_{c}^{[a} \gamma^{b]}+c_{2} \bar{\Sigma}^{a b} \gamma_{c}
$$

The coefficients $c_{1}, c_{2}$ are fixed by the Bianchi identity (3.6) to the values:

$$
c_{1}=2 i, c_{2}=-i
$$

However the other Bianchi identities hold only on-shell, i.e. for $R^{a b}=R^{a}=\Sigma=0$. Then the invariances generated by the Lie derivative:

$$
\begin{aligned}
\delta V^{a} & =\delta_{\text {gauge }} V^{a} \\
\delta \omega^{a b} & =\delta_{\text {gauge }} \omega^{a b}+2 \varepsilon^{c} R^{a b}{ }_{c d} V^{d}+\bar{\theta}^{a b}{ }_{c} \epsilon V^{c}-\bar{\theta}^{a b}{ }_{c} \psi \varepsilon^{c} \\
\delta \psi & =\delta_{\text {gauge }} \psi+2 \varepsilon^{a} \Sigma_{a b} V^{b}
\end{aligned}
$$

are still invariances of the action, but only close on-shell. 
Notice that the origin of supersymmetry is completely algebraic for the Chern-Simons action, while it is geometric (due to superdiffeomorphism invariance of a superspace action) for the rheonomic action.

\section{Off-shell $N=1, d=3$ AdS supergravity}

\subsection{Off-shell degrees of freedom}

The mismatch between the 3 off-shell bosonic degrees of freedom of the dreibein $(d(d-1) / 2$ in $d$ dimensions), and the 4 off-shell degrees of freedom of the gravitino $\left((d-1) 2^{[d / 2]}\right.$ in $d$ dimensions for Majorana or Weyl) can be cured by introducing an extra bosonic d.o.f., here provided by a bosonic 2-form auxiliary field $B$.

\subsection{The extended superAdS algebra}

The algebraic starting point is the FDA (Free Differential Algebra, see [9-11]) that enlarges the $d=3$ superAdS Cartan-Maurer equations to include the auxiliary 2-form field $B$. This extension of the superAdS algebra is possible due to the existence of the $d=3$ cohomology class $\Omega=\bar{\psi} \gamma_{a} \psi V^{a}$, which is closed because of the $d=3$ Fierz identity (A.11).

The FDA yields the definitions of the AdS Lorentz curvature, the supertorsion, the AdS gravitino field strength and the 2-form field strength:

$$
\begin{aligned}
R^{a b} & =d \omega^{a b}-\omega_{c}^{a} \omega^{c b}-\frac{1}{\lambda^{2}} V^{a} V^{b}+\frac{i}{2 \lambda} \bar{\psi} \gamma^{a b} \psi \\
R^{a} & =d V^{a}-\omega_{b}^{a} V^{b}-\frac{i}{2} \bar{\psi} \gamma^{a} \psi \equiv \mathcal{D} V^{a}-\frac{i}{2} \bar{\psi} \gamma^{a} \psi \\
\Sigma & =d \psi-\frac{1}{4} \omega^{a b} \gamma_{a b}-\frac{1}{2 \lambda} V^{a} \gamma_{a} \psi \psi \equiv \mathcal{D} \psi-\frac{1}{2 \lambda} V^{a} \gamma_{a} \psi \\
R^{\otimes} & =d B-\frac{i}{2} \bar{\psi} \gamma^{a} \psi V^{a}+\frac{1}{3 \lambda} V^{a} V^{b} V^{c} \varepsilon_{a b c}
\end{aligned}
$$

The generalized Cartan-Maurer equations are invariant under the rescalings

$$
\omega^{a b} \rightarrow \omega^{a b}, V^{a} \rightarrow u V^{a}, \psi \rightarrow u^{\frac{1}{2}} \psi, B \rightarrow u^{2} B
$$

Taking exterior derivatives of both sides yields the Bianchi identities:

$$
\begin{aligned}
\mathcal{D} R^{a b}+\frac{2}{\lambda^{2}} R^{[a} V^{b]}+\frac{i}{\lambda} \bar{\psi} \gamma^{a b} \Sigma & =0 \\
\mathcal{D} R^{a}+R^{a}{ }_{b} V^{b}-i \bar{\psi} \gamma^{a} \Sigma & =0 \\
\mathcal{D} \Sigma+\frac{1}{4} R^{a b} \gamma_{a b} \psi+\frac{1}{2 \lambda} R^{a} \gamma_{a} \psi-\frac{1}{2 \lambda} V^{a} \gamma_{a} \Sigma & =0 \\
d R^{\otimes}-i \bar{\psi} \gamma^{a} \Sigma V^{a}+\frac{i}{2} \bar{\psi} \gamma^{a} \psi R^{a}-\frac{1}{\lambda} R^{a} V^{b} V^{c} \varepsilon_{a b c} & =0
\end{aligned}
$$

\subsection{Curvature parametrizations}

As in the preceding section, we impose some algebraic constraints on the curvature components to ensure invariance of the spacetime action under local supersymmetry. In this case, 
the presence of the auxiliary field $B$ allows the off-shell closure of the symmetry algebra and now Bianchi identities hold also off-shell. The required parametrization is given by

$$
\begin{aligned}
R^{a b} & =R_{c d}^{a b} V^{c} V^{d}+\bar{\theta}_{c}^{a b} \psi V^{c}+c_{1} f \bar{\psi} \gamma^{a b} \psi \\
R^{a} & =0 \\
\Sigma & =\Sigma_{a b} V^{a} V^{b}-c_{2} f V^{a} \gamma_{a} \psi \\
R^{\otimes} & =f V^{a} V^{b} V^{c} \epsilon_{a b c} \\
d f & =\partial_{a} f V^{a}+\bar{\psi} \Xi
\end{aligned}
$$

with

$$
\bar{\theta}_{c}^{a b}=c_{3} \bar{\Sigma}_{c}^{[a} \gamma^{b]}+c_{4} \bar{\Sigma}^{a b} \gamma_{c}, \quad \Xi^{\alpha}=c_{5} \epsilon^{a b c} \gamma_{a} \Sigma_{b c}
$$

The coefficients $c_{1}, c_{2}, c_{3}, c_{4}, c_{5}$ are fixed by the Bianchi identities to the values:

$$
c_{1}=\frac{3 i}{2}, c_{2}=\frac{3}{2}, c_{3}=2 i, c_{4}=-i, c_{5}=-\frac{i}{3 !}
$$

The $V V V$ component $f$ of $R^{\otimes}$ scales as $f \rightarrow u^{-1} f$, and is identified with the auxiliary scalar superfield of the superspace approach of ref. [6]. Thanks to the presence of the auxiliary field, the Bianchi identities do not imply equations of motion for the spacetime components of the curvatures.

\subsection{The Lagrangian}

The usual building rules of the geometric approach lead to the Lagrangian 3-form

$$
\begin{aligned}
L= & R^{a b} V^{c} \varepsilon_{a b c}+2 i \bar{\psi} \Sigma+\frac{2}{3 \lambda^{2}} V^{a} V^{b} V^{c}-\frac{i}{2 \lambda} \bar{\psi} \gamma^{a b} \psi V^{c} \varepsilon_{a b c}+ \\
& +\alpha\left(f R^{\otimes}-\frac{1}{2} f^{2} V^{a} V^{b} V^{c} \varepsilon_{a b c}\right)
\end{aligned}
$$

The remaining parameter is fixed to $\alpha=6$ by requiring $\iota_{\epsilon} d L^{3 \mid 0}=$ exact, i.e. supersymmetry invariance of the spacetime action. Indeed with $\alpha=6$ we find $d L=0$ on the (off-shell) field configurations satisfying the curvature parametrizations (4.10)-(4.14).

\subsection{Off-shell supersymmetry transformations}

The off-shell closure of the supersymmetry transformations is ensured because the Bianchi identities hold without recourse to the spacetime field equations. The action is invariant under these transformations, given by the Lie derivative of the fields along the fermionic directions:

$$
\begin{aligned}
\delta_{\epsilon} V^{a} & =-i \bar{\psi} \gamma^{a} \epsilon \\
\delta_{\epsilon} \psi & =\mathcal{D} \epsilon-\frac{1}{2 \lambda} V^{a} \gamma_{a} \epsilon+\frac{3}{2} f V^{a} \gamma_{a} \epsilon \\
\delta_{\epsilon} \omega^{a b} & =\bar{\theta}^{a b}{ }_{c} \epsilon V^{c}-3 i f \bar{\psi} \gamma^{a b} \epsilon+\frac{i}{\lambda} \bar{\psi} \gamma^{a b} \epsilon \\
\delta_{\epsilon} B & =-i \bar{\psi} \gamma^{a} \epsilon V^{a} \\
\delta_{\epsilon} f & =\bar{\epsilon} \Xi
\end{aligned}
$$




\subsection{Field equations}

Varying $\omega^{a b}, V^{a}, \psi, B$ and $f$ in the action $S=\int_{M^{3 \mid 2}} L^{3} \wedge \eta_{M^{3}}$ leads to the equations of motion:

$$
\begin{aligned}
R^{a} & =0 \\
R^{a b} & =9 f^{2} V^{a} V^{b}-\frac{6 f}{\lambda} V^{a} V^{b}+\frac{3 i}{2} f \bar{\psi} \gamma^{a b} \psi \\
\Sigma & =-\frac{3}{2} V^{a} \gamma_{a} \psi f \\
d f & =0 \\
R^{\otimes} & =f V^{a} V^{b} V^{c} \varepsilon_{a b c}
\end{aligned}
$$

\section{Equivalence of transformations: trivial gauge transformations}

Here we show that the gauge transformations of super CS action are equivalent to the diffeomorphism transformations on the supergravity counterpart, modulo trivial gauge transformations, i.e. transformations which are proportional to the equations of motion (these transformation were first introduced in [16] in describing quantisation of reducible gauge systems).

Let us start from the CS side: we analyse first the bosonic symmetries and therefore we restric ourselves to a pure gravity theory. From the gauge fields $A$ and $\tilde{A}$ we can obtain the dreibein $V^{a}$ and the spin connection $\omega^{a b}$ as linear combinations of $A$ and $\tilde{A}$. Let us focus on their bosonic gauge transformations:

$$
\delta V^{a}=\mathcal{D} \varepsilon^{a}+\varepsilon^{a b} V_{b}, \delta \omega^{a b}=\mathcal{D} \varepsilon^{a b}
$$

On the other hand, let us see how $V$ transforms under diffeomorphisms and Lorentz symmetries:

$$
\tilde{\delta} V^{a}=\ell_{X} V^{a}+\lambda_{b}^{a} V^{b}, \tilde{\delta} \omega^{a b}=\ell_{X} \omega^{a b}+\mathcal{D} \lambda^{a b}
$$

where $X$ is a vector field and $\lambda_{b}^{a}$ are the Lorentz parameters. We can recast the transformations in (5.2) as follows:

$$
\begin{aligned}
\tilde{\delta} V^{a} & =\iota_{X} V^{a}+\omega^{a}{ }_{b} \wedge V^{b} \\
& =\iota_{X}\left(d V^{a}-\omega^{a}{ }_{b} \wedge V^{b}\right)+\iota_{X}\left(\omega^{a}{ }_{b} \wedge V^{b}\right)+\mathcal{D}\left(\iota_{X} V^{a}\right)+\omega^{a}{ }_{b} \wedge \iota_{X} V^{b}+\lambda^{a}{ }_{b} \wedge V^{b} \\
& =\iota_{X} R^{a}+\iota_{X} \omega^{a}{ }_{b} \wedge V^{b}+\mathcal{D}\left(\iota_{X} V^{a}\right)+\lambda^{a}{ }_{b} \wedge V^{b} \\
& =\iota_{X} R^{a}+\mathcal{D}\left(\iota_{X} V^{a}\right)+\left(\lambda+\iota_{X} \omega\right)^{a}{ }_{b} \wedge V^{b} .
\end{aligned}
$$

We have therefore distinguished three pieces: the first one is written in terms of the torsion $R^{a}$, the second may be identified with $\mathcal{D} \varepsilon^{a}$ if we identify

$$
\iota_{X} V^{a} \equiv \varepsilon^{a} \Longrightarrow X^{\mu} V_{\mu}^{a} \equiv \varepsilon^{a},
$$

and the last term is identified with $\varepsilon$ if we identify

$$
\varepsilon \equiv \lambda+\iota_{X} \omega .
$$


Therefore, the difference between the gauge and Lorentz transformations and the diffeomorphisms and Lorentz transformations is

$$
(\delta-\tilde{\delta}) V^{a}=-\iota_{X} R^{a} .
$$

We can perform the same manipulations of (5.3) for the spin connection as well:

$$
\tilde{\delta} \omega^{a b}=\iota_{X}(d \omega-\omega \wedge \omega)^{a b}+\mathcal{D}\left(\iota_{X} \omega^{a b}\right)+\mathcal{D} \Omega^{a b}=\iota_{X} R^{a b}+\mathcal{D}\left(\lambda+\iota_{X} \omega\right)^{a b}
$$

The first term is written in terms of the curvature $R^{a b}$, while the second term can be identified with (5.2) by setting

$$
\varepsilon \equiv \lambda+\iota_{X} \omega
$$

Again, the difference between gauge + Lorentz and diffeomorphism + Lorentz transformations reads

$$
(\delta-\tilde{\delta}) \omega^{a b}=-\iota_{X} R^{a b} .
$$

Recall that, given the Einstein-Hilbert action, we can recast $R^{a b}$ and $R^{a}$ as

$$
\frac{\delta S}{\delta V^{a}}=\epsilon_{a b c} R^{b c}, \frac{\delta S}{\delta \omega^{a b}}=\epsilon_{a b c} R^{c} .
$$

We can therefore recast (5.6) and (5.9) as

$$
(\delta-\tilde{\delta}) V_{\mu}^{a}=\epsilon^{a b c} X_{\nu} \epsilon_{\mu}^{\nu \rho} \frac{\delta S}{\delta \omega_{\rho}^{b c}}, \quad(\delta-\tilde{\delta}) \omega_{\mu}^{a b}=\epsilon^{a b c} X_{\nu} \epsilon_{\mu}^{\nu \rho} \frac{\delta S}{\delta V_{\rho}^{c}} .
$$

These kind of transformations, defined by a parameter multiplying the equations of motions, are called trivial gauge transformations; any action is invariant under these transformations and they can be cast in the following from

$$
\delta \phi^{A}=\mu^{A B} \frac{\delta S}{\delta \phi^{B}}
$$

for any field $\phi^{A}$ of the model. The gauge parameters $\mu^{A B}$ are local, possibly field-dependent gauge parameters. They are antisymmetric $\mu^{A B}=-\mu^{B A}$ and leave any action invariant

$$
\delta S=\int \delta \phi^{A} \frac{\delta S}{\delta \phi^{A}}=\int \mu^{A B} \frac{\delta S}{\delta \phi^{B}} \frac{\delta S}{\delta \phi^{A}}=0 .
$$

The commutator of any gauge transformation of the theory

$$
\delta_{T} S=T^{A} \frac{\delta S}{\delta \phi^{A}}=0
$$

with trivial gauge transformations (5.12)

$$
\left[\delta_{\mu}, \delta_{T}\right] \phi^{A}=\left(\frac{\delta T^{A}}{\delta \phi^{B}} \mu^{B C}-\frac{\delta T^{C}}{\delta \phi^{B}} \mu^{B A}-T^{A} \frac{\delta \mu^{B C}}{\delta \phi^{B}}\right) \frac{\delta S}{\delta \phi^{C}}
$$


leads again to a trivial gauge transformation. This set of trivial gauge transformations forms a normal (i.e. invariant) subgroup of the full gauge group. They are not physically relevant since: 1) they exist independently of the action; in other words, they do not restrict at all the form of the Lagrangian and no non-trivial Noether identity is associated with them. 2) they imply no degeneracy of the action and in the Hamiltonian formalism, there is no corresponding constraint. Actually, the conserved charges associated with those gauge transformation, when rewritten as phase space functions, vanish identically. 3) The trivial gauge transformations vanish on-shell, i.e., do not map solutions of the equations of motion on new, different solutions. 4) There is accordingly no need for a "gauge fixing". On this basis, it is natural to disregard them and, being a normal subgroup, this is well defined procedure [17-20].

Notice that (5.6) and (5.8), once inserted into the action, give rise to

$$
\iota_{X}\left(R^{a} R^{b c} \epsilon_{a b c}\right)=\iota_{X}(d L)=0,
$$

since $d L=0$ being $L$ a 3 -form in a 3 -dimensional manifold.

Now, we proceed with the local supersymmetry transformations for $d=3$ supergravity. We write the susy transformations as Lie derivatives along fermionic directions:

$$
\begin{aligned}
\tilde{\delta} V^{a} & =\ell_{\epsilon} V^{a}=d \iota_{\epsilon} V^{a}+\iota_{\epsilon} d V^{a}=\iota_{\epsilon} R^{a}+\iota_{\epsilon}(\omega \wedge V)^{a}+\iota_{\epsilon}\left(\frac{i}{2} \bar{\psi} \gamma^{a} \psi\right)= \\
& =\iota_{\epsilon} R^{a}+\left[\iota_{\epsilon}(\omega) V\right]^{a}+i \epsilon \gamma^{a} \psi=\iota_{\epsilon} R^{a}+i \epsilon \gamma^{a} \psi,
\end{aligned}
$$

where we have used $\iota_{\epsilon} V^{a}=0=\iota_{\epsilon} \omega^{a b}$ since they have no "legs" in the fermionic directions. We can repeat the same manipulations for $\omega$ and $\psi$ in order to obtain

$$
\tilde{\delta}_{\epsilon} \omega^{a b}=\iota_{\epsilon} R^{a b}-\frac{1}{\lambda} \bar{\epsilon} \gamma^{a b} \psi, \tilde{\delta}_{\epsilon} \psi=\iota_{\epsilon} \Sigma+\mathcal{D} \epsilon+V^{a} \gamma_{a} \epsilon
$$

We can now compute the difference between the supersymmetry transformations and the superdiffeomorphisms along fermionic directions:

$$
\begin{gathered}
(\delta-\tilde{\delta}) V^{a}=-\iota_{\epsilon} R^{a} \\
(\delta-\tilde{\delta}) \omega^{a b}=-\iota_{\epsilon} R^{a b} \\
(\delta-\tilde{\delta}) \psi=-\iota_{\epsilon} \Sigma
\end{gathered}
$$

Notice that once these transformations are inserted into the Lagrangian we obtain

$$
(\delta-\tilde{\delta}) L=\iota_{\epsilon}\left(R^{a} R^{b c} \epsilon_{a b c}+2 i \bar{\Sigma} \Sigma\right)=\iota_{\epsilon} d L
$$

which is the contraction of the exterior derivative of the Lagrangian discussed in section 3.3. As mentioned in section 4.4, these trivial gauge transformations vanish identically if the Lagrangian is closed, which is the condition that we discuss in the following section while constructing super Chern-Simons theory on supergroups. 


\section{Supersymmetric Achucarro-Townsend model}

The model discussed up to this point shows a gauge supersymmetry which is translated into a local supersymmetry in the supergravity re-interpretation. Now, we would like to choose a different path and before rewriting the Achucarro-Townsend Chern-Simons theory in terms of vielbein $V^{a}$, the spin connection $\omega^{a b}$ and the gravitino $\psi$, we promote it to a worldvolume supersymmetric Chern-Simons model. For that, we introduce the worldvolume vielbein $e^{a}$ and a worldvolume gravitino $\chi^{\alpha}$ (where the index $\alpha=1,2$ denotes the two components of the wordvolume spinor) such that

$$
d e^{a}=\bar{\chi} \gamma^{a} \chi, \quad d \chi^{\alpha}=0
$$

i.e. assume a flat worldvolume. To translate the CS action given in (2.1) into a worldvolume supersymmetric model, we recall the properties of the $\operatorname{OSp}(1 \mid 2)$ super algebra (we consider here only the $O S p$ part of the CS action (2.1)); it can be described in terms of the generators $T_{a}, Q_{\alpha}$ with the commutators

$$
\left[T_{a}, T_{b}\right]=i \epsilon_{a b}{ }^{c} T_{c}, \quad\left[T_{a}, Q_{\alpha}\right]=\gamma_{a \alpha}^{\beta} Q_{\beta}, \quad\left\{Q_{\alpha}, Q_{\beta}\right\}=2 i \gamma_{\alpha \beta}^{a} T_{a}
$$

The indices are $a=1, \ldots, 3$ and $\alpha, \beta=1, \ldots, 2$. The invariant tensors are defined as follows

$$
\operatorname{Str}\left(T_{a} T_{b}\right)=\eta_{a b}, \quad \operatorname{Str}\left(Q_{\alpha} Q_{\beta}\right)=\epsilon_{\alpha \beta}, \quad \operatorname{Str}\left(T_{a} Q_{\alpha}\right)=0 .
$$

The first one is symmetric $\eta_{a b}=\eta_{b a}$ and the second one is anti-symmetric $\epsilon_{\alpha \beta}=-\epsilon_{\beta \alpha}$. They are both invertible.

The supergroup connection $\boldsymbol{\Omega}$ is defined as (2.2)

$$
\boldsymbol{\Omega}=A^{a} T_{a}+\psi^{\alpha} Q_{\alpha}
$$

To respect the statistics of $\boldsymbol{\Omega}, A^{a}$ is an anticommuting connection (bosonic 1-form) and $\psi^{\alpha}$ is a commuting connection (fermionic 1-form). Promoting both $A^{a}$ and $\psi^{\alpha}$ to (1|0)superforms (for a discussion on forms on supermanifolds see e.g. [12-15]), they read

$$
A^{a}=A_{b}^{a} e^{b}+A_{\beta}^{a} \chi^{\beta}, \quad \psi^{\alpha}=\psi_{b}^{\alpha} e^{b}+\psi_{\beta}^{\alpha} \chi^{\beta},
$$

where $A_{b}^{a}$ and $\psi_{\beta}^{\alpha}$ are commuting superfields and $A_{\alpha}^{a}$ and $\psi_{b}^{\alpha}$ anticommuting superfields. The field strengths are defined as (for simplicity we set $\lambda=1$ in the following and to distinguish them from the $x$-dependent $\boldsymbol{\Omega}$ connection, we will rename the superspacedependent connection with $\mathcal{A}$ )

$$
\begin{aligned}
\mathcal{F} & =d \mathcal{A}-[\mathcal{A}, \mathcal{A}\}, \\
F^{a} & =d A^{a}+\epsilon_{b c}^{a} A^{b} \wedge A^{c}-\frac{1}{2} \bar{\psi} \gamma^{a} \psi, \\
F^{\alpha} & =d \psi^{\alpha}-A^{a}\left(\gamma_{a} \psi\right)^{\alpha} .
\end{aligned}
$$


Notice that (6.6) coincide with the $\operatorname{OSp}(1 \mid 2)$ part of (2.4) and (2.6) but hold on the whole superspace. Their rheonomic parameterizations are the following

$$
\begin{aligned}
\mathcal{F} & =\mathcal{F}_{a b} e^{a} \wedge e^{b}+\left(\mathcal{W} \gamma_{a} \chi\right) \wedge e^{a}, \\
\nabla \mathcal{W}_{\alpha} & =e^{a} \nabla_{a} \mathcal{W}_{\alpha}-\frac{1}{4}\left(\gamma^{a b} \chi\right) \mathcal{F}_{a b}, \\
F^{a} & =F_{b c}^{a} e^{b} \wedge e^{c}+\left(\Xi^{a} \gamma_{b} \chi\right) \wedge e^{b}, \\
\nabla \Xi^{a} & =e^{b} \nabla_{b} \Xi^{a}-\left(\frac{1}{4} \gamma^{b c} \chi\right) F_{b c}^{a}, \\
F^{\alpha} & =F_{a b}^{\alpha} e^{a} \wedge e^{b}+\left(G^{\alpha} \gamma_{a} \chi\right) \wedge e^{a}, \\
\nabla G^{\alpha} & =e^{a} \nabla_{a} G^{\alpha}-\left(\frac{1}{4} \gamma^{a b} \chi\right) F_{a b}^{\alpha},
\end{aligned}
$$

and satisfy the Bianchi identities

$$
d \mathcal{F}+[\mathcal{A}, \mathcal{F}\}=0
$$

The superfield $\mathcal{W}$ is a 0 -form spinor superfield and its components are defined as

$$
\mathcal{W}=\Xi^{a} T_{a}+G^{\alpha} Q_{\alpha}
$$

The fields $\Xi^{a}$ (anticommuting) and $G^{\alpha}$ (commuting) are 0-form woldvolume spinors with indices in the vector and spinor representation of $\mathrm{SO}(1,2)$, respectively. Their covariant derivatives are defined as follows

$$
\begin{aligned}
\nabla \mathcal{W} & =d \mathcal{W}+[\mathcal{A}, \mathcal{W}\} \\
(\nabla \Xi)^{a} & =d \Xi^{a}+\epsilon^{a}{ }_{b c} A^{b} \Xi^{c}+\gamma_{\alpha \beta}^{a} G^{\alpha} \psi^{\beta}, \\
(\nabla G)^{\alpha} & =d G^{\alpha}-\gamma_{a \beta}^{\alpha}\left(A^{a} G^{\beta}+\Xi^{a} \psi^{\beta}\right) .
\end{aligned}
$$

The supersymmetry transformations can be easily obtained by using the rheonomic parameterization (6.7)

$$
\begin{aligned}
& \delta_{\epsilon} A^{a}=\mathcal{L}_{\epsilon} A^{a}=\iota_{\epsilon} F^{a}+\nabla \Lambda^{a}=\left(\Xi^{a} \gamma_{b} \epsilon\right) e^{b}+\nabla \Lambda^{a}, \\
& \delta_{\epsilon} \psi^{\alpha}=\mathcal{L}_{\epsilon} \psi^{\alpha}=\iota_{\epsilon} F^{\alpha}+\nabla \Lambda^{\alpha}=\left(G^{\alpha} \gamma_{a} \epsilon\right) e^{a}+\nabla \Lambda^{\alpha},
\end{aligned}
$$

where $\Lambda^{a}=\iota_{\epsilon} A^{a}$ and $\Lambda^{\alpha}=\iota_{\epsilon} \psi^{\alpha}$. The fields $\Xi^{a}$ and $G^{\alpha}$ are the superpartners of $A^{a}$ and $\psi^{\alpha}$. No auxiliary field is needed and the matching is achieved both off-shell and on-shell. All the symmetries close off shell because gauge symmetries close by construction in SCS theories and the supersymmetries close off-shell in $d=3$ supergravity.

The action invariant under both gauge and worldvolume symmetries is given by [21]

$$
\mathcal{L}^{(3 \mid 0)}=\operatorname{Str}\left(\mathcal{A} d \mathcal{A}-\frac{2}{3} \mathcal{A}[\mathcal{A}, \mathcal{A}\}+\mathcal{W}_{\alpha} \mathcal{W}_{\beta} \epsilon^{\alpha \beta} \operatorname{Vol}^{3}\right)
$$

$\mathrm{Vol}^{3}$ is the volume form on the three-dimensional manifold, $\mathrm{Vol}^{3}=\frac{1}{3 !} \epsilon_{a b c} e^{a} e^{b} e^{c} .{ }^{5}$ We use the notation $\mathcal{W} \cdot \mathcal{W}=\mathcal{W}^{\alpha} \epsilon_{\alpha \beta} \mathcal{W}^{\beta}$.

\footnotetext{
${ }^{5} d \mathrm{Vol}^{3}=\frac{1}{2} \epsilon_{a b c} \bar{\chi} \gamma^{a} \chi e^{b} e^{c}=\frac{1}{2} \rho_{\alpha} \epsilon^{\alpha \beta} \rho_{\beta}$ with $\rho_{\alpha}=\left(V^{a} \gamma_{a} \psi\right)_{\alpha}$ and $d \rho_{\alpha}=0$.
} 
Computing the super trace explicitly we have

$$
\begin{aligned}
\mathcal{L}^{(3 \mid 0)}= & \left(\eta_{a b} A^{a} d A^{b}-\frac{2}{3} \epsilon_{a b c} A^{a} A^{b} A^{c}+\eta_{a b} \Xi^{a} \cdot \Xi^{b} \mathrm{Vol}^{3}\right. \\
& \left.+\epsilon_{\alpha \beta} \bar{\psi}^{\alpha} d \psi^{\beta}+A^{a} \bar{\psi} \gamma_{a} \psi+\epsilon_{\alpha \beta} G^{\alpha} \cdot G^{\beta} \mathrm{Vol}^{3}\right) .
\end{aligned}
$$

The Chern-Simons gauge symmetries are given by

$$
\delta \mathcal{A}=d C+[\mathcal{A}, C\}, \quad \delta \mathcal{W}=[\mathcal{W}, C\}, \quad C=C^{a} T_{a}+C^{\alpha} Q_{\alpha},
$$

where $C$ is the gauge superfield parameter with values in the super-Lie algebra.

Let us compute the differential of $\mathcal{L}^{(3 \mid 0)}$

$$
\begin{aligned}
d \mathcal{L}^{(3 \mid 0)}= & \operatorname{Str}\left(\mathcal{F} \wedge \mathcal{F}+2 d \mathcal{W} \cdot \mathcal{W} \operatorname{Vol}^{3}+\frac{1}{2} \mathcal{W}^{2} \epsilon_{a b c}\left(\bar{\chi} \gamma^{a} \chi\right) e^{b} e^{c}\right) \\
= & \operatorname{Str}\left(2 \mathcal{F}_{a b} e^{a} \wedge e^{b}\left(\mathcal{W} \gamma_{c} \chi\right) \wedge e^{c}+\left(\mathcal{W} \gamma_{a} \chi\right) \wedge e^{a}\left(\mathcal{W} \gamma_{b} \chi\right) \wedge e^{b}\right. \\
& \left.+2-\frac{1}{4}\left(\mathcal{W} \gamma^{a b} \chi\right) \operatorname{Vol}^{3} \mathcal{F}_{a b}+\frac{1}{2} \mathcal{W}^{2} \epsilon_{a b c}\left(\bar{\chi} \gamma^{a} \chi\right) e^{b} e^{c}\right)=0
\end{aligned}
$$

which agrees with the fact that the rheonomic Lagrangian is closed if there are auxiliary fields or if they are not needed. We get also the interesting equation

$$
\operatorname{Str}(\mathcal{F} \wedge \mathcal{F})=-d\left(\operatorname{Str}\left(\mathcal{W}^{2}\right) \operatorname{Vol}^{3}\right)
$$

Both members are gauge invariant under super gauge transformations. Since we have $d \mathcal{L}^{(3 \mid 0)}=0$, this implies

$$
\delta d \mathcal{L}^{(3 \mid 0)}=0 \Longrightarrow \delta \mathcal{L}^{(3 \mid 0)}=d \Omega_{1}^{(2 \mid 0)}
$$

where $\Omega_{1}^{(2 \mid 0)}$ is a $(2 \mid 0)$ form. In turn, this implies that the action is gauge invariant up to boundary terms.

We can now add the other part of the supergroup: we consider as in the previous sections $\operatorname{OSp}(1 \mid 2) \times \operatorname{Sp}(2)$ and this rerequires to subtract a super Chern-Simons sction (i.e. with worldvolume supersymmetry) to the action (6.13):

$$
\begin{aligned}
\mathcal{L}^{(3 \mid 0)}= & \left(\eta_{a b} A^{a} d A^{b}+\frac{2}{3} \epsilon_{a b c} A^{a} A^{b} A^{c}+\eta_{a b} \Xi^{a} \cdot \Xi^{b} \mathrm{Vol}^{3}\right. \\
& \left.+\epsilon_{\alpha \beta} \bar{\psi}^{\alpha} d \psi^{\beta}+A^{a} \bar{\psi} \gamma_{a} \psi+\epsilon_{\alpha \beta} G^{\alpha} \cdot G^{\beta} \mathrm{Vol}^{3}\right)+ \\
& -\left(\eta_{a b} \tilde{A}^{a} d \tilde{A}^{b}+\frac{2}{3} \epsilon_{a b c} \tilde{A}^{a} \tilde{A}^{b} \tilde{A}^{c}+\eta_{a b} \tilde{\Xi}^{a} \cdot \tilde{\Xi}^{b} \mathrm{Vol}^{3}\right) .
\end{aligned}
$$

Again the Lagrangian is closed because of the presence of the "auxiliary fields" $\Xi, G$ and $\tilde{\Xi}$ and its exterior derivative takes the form (using the parametrisations (6.7) when calculating $d \Xi)$

$$
\begin{aligned}
0=d \mathcal{L}^{(3 \mid 0)}= & F \wedge F-\tilde{F} \wedge \tilde{F}+(\nabla \psi)^{2}-\frac{1}{2} \operatorname{Vol}^{3} \chi \gamma^{c d} \eta_{a b}\left(\Xi^{a} F_{c d}^{b}-\tilde{\Xi}^{a} \tilde{F}_{c d}^{b}\right)+ \\
& +\eta_{a b}\left(\Xi^{a} \Xi^{b}-\tilde{\Xi}^{a} \tilde{\Xi}^{b}\right) 3 \epsilon_{a b c} \bar{\chi} \gamma^{a} \chi e^{b} e^{c}-\frac{1}{2} \epsilon_{\alpha \beta} \gamma^{a b} \chi F_{a b}^{\alpha} G^{\beta} \operatorname{Vol}^{3}+ \\
& +\epsilon_{\alpha \beta} G^{\alpha} G^{\beta} 3 \epsilon_{a b c} \bar{\chi} \gamma^{a} \chi e^{b} e^{c} .
\end{aligned}
$$


If we set $\Xi=\tilde{\Xi}$, we have that the first term of the second line vanishes, while the last term of the first line becomes

$$
\frac{1}{2} \operatorname{Vol}^{3} \chi \gamma^{c d} \eta_{a b} \Xi^{a}\left(F_{c d}^{b}-\tilde{F}_{c d}^{b}\right)
$$

This term vanishes if

$$
F_{a b}=\tilde{F}_{a b}+\bar{\chi} \gamma_{a b} \chi
$$

because of the Fierz identities (A.11). Once we identify the difference of the gauge fields with the dreibein $V$ as in (2.9), (6.21) is exactly the vanishing torsion-condition, as we can see from (4.2):

$$
F_{a b}=\tilde{F}_{a b}+\bar{\chi} \gamma_{a b} \chi \Longrightarrow R^{a}=0 .
$$

Consider the counting of the degrees of freedom. For the fields of starting Lagrangian counting is

$$
\begin{array}{cccc}
A: & 9-3=6, & \Xi: & 6 \\
\Psi: & 6-2=4, & G: & 4 \\
\tilde{A}: & 9-3=6, & \tilde{\Xi}: & 6 \\
\hline 8 & & 8
\end{array}
$$

The matching is established by construction since the Lagrangian itself has been built by associating a partner to each field. The condition $\Xi=\tilde{\Xi}$ removes 6 d.o.f. on the right, but since it implies the torsionless condition it removes 3 d.o.f. on the left as well. Now, the counting is 5 vs 2 . The matching is established by requiring that out of the 4 d.o.f. of the auxiliary field $G$ only 1 is nontrivial; this is obtained via the parametrisation

$$
G_{\alpha^{\prime}}^{\alpha}=G \delta_{\alpha^{\prime}}^{\alpha}
$$

This field $G$ can be identified with auxiliary field $f$ of section 4 .

\section{Supersymmetry}

We clarify some issues regarding supersymmetry matching of d.o.f. We also point out that here we do not take a supergravity interpretation of Chern-Simons theory, but we explore the matching of d.o.f.'s as in a pure Chern-Simons gauge theory. Later, we discuss its supergravity interpretation and discuss different supergroups.

First consider the supersymmetry on the worlvolume. In that case, for each gauge field $A^{a}$ there is a corresponding spinor field $\Xi^{a}$. The matching off-shell is achieved by noting that, because of the gauge symmetry $\delta A^{a}=\nabla C^{a}$, we can remove one degree of freedom (for each generator of the Lie algebra) from $A^{a}$. Then, the remaining d.o.f.'s matche with those of the gauginos $\Xi^{\alpha}$. On the other hand, using the equations of motion, we find that there are no propagating d.o.f. for the gauge fields, since their field strength vanishes, and for the gauginos which have algebraic (non dynamical) equations.

The same argument applies also in the case of the gauge fields $\psi^{\alpha}$ associated to the supercharges. Gauge symmetry removes one d.o.f. from $\psi^{\alpha}$ and the remaining d.o.f. match those of $G^{\alpha}$. Again, on-shell there are no propagating degrees of freedom and the matching is trivial. 
We consider now a different type of matching. We would like to compare the d.o.f.'s of the gauge fields $A^{a}$ with those of $\psi^{\alpha}$. They are both gauge fields and off-shell correspond $2 \times$ bosonic generators and $2 \times$ fermionic generators of the gauge supergroup. Consider then the supergroup $O S p(p \mid q)$. The counting of bosonic vs fermionic generators gives

$$
\left(\frac{p(p-1)}{2}+\frac{q(q+1)}{2} \mid p q\right)
$$

and therefore the matching is achieved when $p=q$ or $p=q+1$. In that case, the bosonic and fermionic d.o.f.'s match. For example the case $q=2$ and $p=2$ or $p=3$ are example of $d=3$ supergravities already known in the literature. However, in general, the matching is not achieved.

If $p \neq q$ we have to follow a different path. Assuming that $p>q$, then we have that the super coset $O S p(p \mid q) / \mathrm{SO}(p-q)$ has the same number of bosonic and fermionic generators $(p q \mid p q)$. On the other side, if $p<q$, we find that the supercoset $O S p(p \mid q) / \operatorname{Sp}(q-p)$ has the same number of bosonic and fermionic generators. Lastly, we have the case $p=1$ and $q=2 r$. In that case, the coset with the matching is $O S p(1 \mid 2 r) / \mathrm{SO}(r, r)$.

We can distinguish the bosonic gauge fields $A^{a}$ between those with the index $I=1, \ldots, p q$ (the number of fermionic generators) $A^{I}$ and those belonging to the subgroups $\mathrm{SO}(p-q)$, or $\mathrm{Sp}(q-p)$ or $\mathrm{SO}(r, r)$ (depending on $p>q, p<q$ or $p=1, q=2 r$ ) $A^{i}$ where $i$ runs over the generators of the subgroup). Then, the field strengths can be divided as follows

$$
F^{I}=d A^{I}+f_{i J}^{I} A^{i} \wedge A^{J}=\nabla A^{I}, \quad F^{i}=d A^{i}+f_{I J}^{i} A^{I} \wedge A^{J}+f_{j k}^{i} A^{j} \wedge A^{k} .
$$

The torsion condition the equation

$$
F^{I}=0
$$

can be solved in terms of $A^{i}$, the gauge fields of the subgroup. In this way, by going (partially) on-shell with those degrees of freedom, we achieve the off-shell matching for the remaining d.o.f.'s. Once this equation is solved in terms of $A^{i}$, we can reinstate them in the rest of the action and derive the corresponding equations of motion. Through the Bianchi identities, we have that

$$
\nabla F^{I}=f_{i J}^{I} F^{i} \wedge A^{J}
$$

and therefore, imposing $F^{I}=0$, we find a condition on $A^{I}$ (this is analogous to impose the vanishing of the torsion in general relativity, solving the spin connection in terms of the vielbein; then, this implies the condition $E \wedge R=0$ for the curvature). This corresponds to the reduced holonomy

$$
f_{i J}^{I} F^{i} \wedge A^{J}=0
$$

Even in this reduced holonomy situation, eq. (7.3) is not always solvable. Indeed, counting the independent contained in (7.3) we have that the index $I$ runs from 1 to $p q$, but they are 2 -form equations which have $p q(p q-1) / 2$ independent components. The unknowns given by the gauge fields $A^{i}$ are 1-form (with $p q$ components for each value of the index $i$ ), 
therefore there are $p q(p-q)^{2}(p-q-1) / 2$ unknowns. To solve the equations we need the matching

$$
p^{2} q^{2}(p q-1)=p q(p-q)(p-q-1)
$$

which can be achieved only if $p=1, \forall q$, or $q=-1, \forall p$ and $q=p /(p+1), \forall p \neq-1$. However, $p$ and $q$ must be positive integers, this excludes the last two solutions. The remaining one, $p=1$, is the only possible case for any $q$. This corresponds to the case $\operatorname{OSp}(1 \mid 2 r)$ with the subgroup $\mathrm{SO}(r, r)$ which is a subgroup of $\mathrm{Sp}(2 r)$. In that case we can solve the equation (7.3) in terms of $A^{i}$. We have the interesting cases $\mathrm{OSp}(1 \mid 2) / \mathrm{SO}(1,1)$, $\operatorname{OSp}(1 \mid 4) / \mathrm{SO}(2,2), \operatorname{OSp}(1 \mid 6) / \mathrm{SO}(3,3)$ and the $\operatorname{OSp}(1 \mid 32) / \mathrm{SO}(16,16)$.

\section{Conclusions and outlook}

In this note we have clarified the issues regarding the relation between the AchucarroTonwsend supergravity models and the group manifold approach to the same theory. In the first case, the gauge symmetry is promoted to a super gauge symmetry and therefore closes off-shell. In the second case, the supersymmetry closes off-shell only after the introduction of auxiliary fields. In the second part of the paper, we construct a double supersymmetric version with worldvolume and gauge supersymmetry and discuss how supergravity can be retrieved. The present work prepares the way to construct AchucarroTonwsend supergravities with extended supersymmetries corresponding to orthosymplectic groups $\operatorname{OSp}(p \mid 2) \times \operatorname{OSp}(q \mid 2)$. Only few cases are studied (see for example [22-32]) in the superspace language, but not in the group manifold approach. In the latter, the question of auxiliary fields has never been tackled.

\section{A Gamma matrices in $D=3$}

We summarize in this appendix our gamma matrix conventions in $D=3$.

$$
\begin{aligned}
& \gamma_{0}=\left(\begin{array}{cc}
i & 0 \\
0 & -i
\end{array}\right), \quad \gamma_{1}=\left(\begin{array}{ll}
0 & 1 \\
1 & 0
\end{array}\right), \quad \gamma_{2}=\left(\begin{array}{cc}
0 & -i \\
i & 0
\end{array}\right) \\
& \eta_{a b}=(-1,1,1), \quad\left\{\gamma_{a}, \gamma_{b}\right\}=2 \eta_{a b}, \quad\left[\gamma_{a}, \gamma_{b}\right]=2 \gamma_{a b}=-2 \varepsilon_{a b c} \gamma^{c}, \\
& \varepsilon_{012}=-\varepsilon^{012}=1 \text {, } \\
& \gamma_{a}^{\dagger}=\gamma_{0} \gamma_{a} \gamma_{0}, \quad \gamma_{a}^{T}=-C \gamma_{a} C^{-1}, \quad C^{T}=-C, \quad C^{2}=\mathbf{1}
\end{aligned}
$$

\section{A.1 Useful identities}

$$
\begin{aligned}
\gamma_{a} \gamma_{b} & =\gamma_{a b}+\eta_{a b}=-\varepsilon_{a b c} \gamma^{c}+\eta_{a b} \\
\gamma_{a b} \gamma_{c} & =\eta_{b c} \gamma_{a}-\eta_{a c} \gamma_{b}-\varepsilon_{a b c} \\
\gamma_{c} \gamma_{a b} & =\eta_{a c} \gamma_{b}-\eta_{b c} \gamma_{a}-\varepsilon_{a b c} \\
\gamma_{a} \gamma_{b} \gamma_{c} & =\eta_{a b} \gamma_{c}+\eta_{b c} \gamma_{a}-\eta_{a c} \gamma_{b}-\varepsilon_{a b c} \\
\gamma^{a b} \gamma_{c d} & =-4 \delta_{[c}^{[a} \gamma_{d]}^{b]}-2 \delta_{c d}^{a b}
\end{aligned}
$$

where $\delta_{c d}^{a b}=\frac{1}{2}\left(\delta_{c}^{a} \delta_{d}^{b}-\delta_{d}^{a} \delta_{c}^{b}\right)$, and index antisymmetrizations in square brackets have weight 1 . 


\section{A.2 Fierz identity for two Majorana one-forms}

$$
\psi \bar{\psi}=\frac{1}{2}\left(\bar{\psi} \gamma^{a} \psi\right) \gamma_{a}
$$

As a consequence

$$
\gamma_{a} \psi \bar{\psi} \gamma^{a} \psi=0
$$

\section{Acknowledgments}

This work has been partially supported by Università del Piemonte Orientale research funds. We would like to thank L. Andrianopoli and M. Trigiante for useful discussions on supergravity.

Open Access. This article is distributed under the terms of the Creative Commons Attribution License (CC-BY 4.0), which permits any use, distribution and reproduction in any medium, provided the original author(s) and source are credited.

\section{References}

[1] A. Achucarro and P.K. Townsend, A Chern-Simons Action for Three-Dimensional anti-de Sitter Supergravity Theories, Phys. Lett. B 180 (1986) 89 [INSPIRE].

[2] L. Castellani, R. Catenacci and P.A. Grassi, The Integral Form of Supergravity, JHEP 10 (2016) 049 [arXiv : 1607.05193] [InSPIRE].

[3] J. Zanelli, Introductory lectures on Chern-Simons theories, AIP Conf. Proc. 1420 (2012) 11.

[4] J. Zanelli, Lecture notes on Chern-Simons (super-)gravities. Second edition (February 2008), in Proceedings, 7th Mexican Workshop on Particles and Fields (MWPF 1999), Merida, Mexico, 10-17 November 1999 (2005) [hep-th/0502193] [INSPIRE].

[5] D.Z. Freedman and A. Van Proeyen, Supergravity, Cambridge University Press (2012) [INSPIRE].

[6] F. Ruiz Ruiz and P. van Nieuwenhuizen, Lectures on supersymmetry and supergravity in $(2+1)$-dimensions and regularization of supersymmetric gauge theories, in Tlaxcala (1996), Recent developments in gravitation and mathematical physics, Tlaxcala, Mexico, 1-7 December 1996 [INSPIRE].

[7] N. Marcus and J.H. Schwarz, Three-Dimensional Supergravity Theories, Nucl. Phys. B 228 (1983) 145 [INSPIRE].

[8] S.J. Gates Jr., M.T. Grisaru, M. Roček and W. Siegel, Superspace Or One Thousand and One Lessons in Supersymmetry, Front. Phys. 58 (1983) 1 [hep-th/0108200] [INSPIRE].

[9] L. Castellani, R. D'Auria and P. Fre, Supergravity and superstrings: A Geometric perspective. Vol. 1: Mathematical foundations, World Scientific, Singapore, pp. 1-603 (1991) [INSPIRE].

[10] L. Castellani, R. D'Auria and P. Fre, Supergravity and superstrings: A Geometric perspective. Vol. 2: Supergravity, World Scientific, Singapore, pp. 607-1371 (1991) [INSPIRE].

[11] L. Castellani, R. D'Auria and P. Fre, Supergravity and superstrings: A Geometric perspective. Vol. 3: Superstrings, World Scientific, Singapore, pp. 1375-2162 (1991) [INSPIRE].

[12] L. Castellani, R. Catenacci and P.A. Grassi, Supergravity Actions with Integral Forms, Nucl. Phys. B 889 (2014) 419 [arXiv: 1409.0192] [INSPIRE]. 
[13] L. Castellani, R. Catenacci and P.A. Grassi, The Geometry of Supermanifolds and New Supersymmetric Actions, Nucl. Phys. B 899 (2015) 112 [arXiv: 1503. 07886] [INSPIRE].

[14] L. Castellani, R. Catenacci and P.A. Grassi, Super Quantum Mechanics in the Integral Form Formalism, Annales Henri Poincaré 19 (2018) 1385 [arXiv:1706.04704] [InSPIRE].

[15] L. Castellani, R. Catenacci and P.A. Grassi, Wess-Zumino and Super Yang-Mills Theories in $D=4$ Integral Superspace, JHEP 05 (2018) 040 [Erratum ibid. 07 (2018) 175] [arXiv: 1711.07194] [INSPIRE].

[16] I.A. Batalin and G.A. Vilkovisky, Quantization of Gauge Theories with Linearly Dependent Generators, Phys. Rev. D 28 (1983) 2567 [Erratum ibid. D 30 (1984) 508] [InSPIRE].

[17] M. Henneaux and C. Teitelboim, Quantization of gauge systems, Princeton University Press, Princeton, U.S.A. (1992) [INSPIRE].

[18] E. Witten, (2+1)-Dimensional Gravity as an Exactly Soluble System, Nucl. Phys. B 311 (1988) 46 [INSPIRE].

[19] E. Witten, Three-Dimensional Gravity Revisited, arXiv:0706.3359 [INSPIRE].

[20] A. Achucarro and P.K. Townsend, Extended Supergravities in $d=(2+1)$ as Chern-Simons Theories, Phys. Lett. B 229 (1989) 383 [inSPIRE].

[21] P.A. Grassi and C. Maccaferri, Chern-Simons Theory on Supermanifolds, JHEP 09 (2016) 170 [arXiv: 1606.06609 ] [INSPIRE].

[22] S.M. Kuzenko, U. Lindström and G. Tartaglino-Mazzucchelli, Off-shell supergravity-matter couplings in three dimensions, JHEP 03 (2011) 120 [arXiv:1101.4013] [INSPIRE].

[23] S.M. Kuzenko and G. Tartaglino-Mazzucchelli, Three-dimensional $N=2$ (AdS) supergravity and associated supercurrents, JHEP 12 (2011) 052 [arXiv:1109.0496] [INSPIRE].

[24] S.M. Kuzenko, U. Lindström and G. Tartaglino-Mazzucchelli, Three-dimensional $(p, q)$ AdS superspaces and matter couplings, JHEP 08 (2012) 024 [arXiv:1205.4622] [INSPIRE].

[25] D. Butter, S.M. Kuzenko, J. Novak and G. Tartaglino-Mazzucchelli, Conformal supergravity in three dimensions: New off-shell formulation, JHEP 09 (2013) 072 [arXiv:1305.3132] [INSPIRE].

[26] D. Butter, S.M. Kuzenko, J. Novak and G. Tartaglino-Mazzucchelli, Conformal supergravity in three dimensions: Off-shell actions, JHEP 10 (2013) 073 [arXiv: 1306.1205] [INSPIRE].

[27] S.M. Kuzenko, U. Lindström, M. Roček, I. Sachs and G. Tartaglino-Mazzucchelli, Three-dimensional $\mathcal{N}=2$ supergravity theories: From superspace to components, Phys. Rev. D 89 (2014) 085028 [arXiv:1312.4267] [INSPIRE].

[28] P. Concha, $N$-extended Maxwell supergravities as Chern-Simons theories in three spacetime dimensions, Phys. Lett. B 792 (2019) 290 [arXiv:1903.03081] [InSPIRE].

[29] P. Concha, D.M. Peñafiel and E. Rodríguez, On the Maxwell supergravity and flat limit in $2+1$ dimensions, Phys. Lett. B $\mathbf{7 8 5}$ (2018) 247 [arXiv:1807.00194] [INSPIRE].

[30] P.K. Concha, O. Fierro and E.K. Rodríguez, Inönü-Wigner contraction and D=2+1 supergravity, Eur. Phys. J. C 77 (2017) 48 [arXiv:1611.05018] [InSPIRE].

[31] P.S. Howe, J.M. Izquierdo, G. Papadopoulos and P.K. Townsend, New supergravities with central charges and Killing spinors in (2+1)-dimensions, Nucl. Phys. B 467 (1996) 183 [hep-th/9505032] [INSPIRE].

[32] J.A. de Azcarraga and J.M. Izquierdo, $(p, q) D=3$ Poincaré supergravities from Lie algebra expansions, Nucl. Phys. B 854 (2012) 276 [arXiv:1107.2569] [INSPIRE]. 